\title{
The effects of rate sensitivity and plastic potential surface curvature on plastic flow localization in porous solids
}

\author{
H.-Y. JEONG and J. PAN \\ Mechanical Engineering and Applied Mechanics, The University of Michigan, Ann Arbor, Michigan 48109, USA
}

Received 30 July 1990; accepted 22 April 1991

\begin{abstract}
Plastic flow localization in porous elastic-viscoplastic solids is analyzed with an emphasis on the effects of material rate sensitivity and plastic potential surface curvature. The effect of rate sensitivity is included in a material model that accounts for a change of yield surface curvature in a rate-insensitive porous ductile solid. Shear band formation under plane strain and axisymmetric tension, and localized necking in biaxially stretched sheets are analyzed by using the present material model. The results illustrate the interactions of the effects of void nucleation and growth, material rate sensitivity and plastic potential surface curvature on plastic flow localization. The effects of nonproportional straining paths on localized necking in thin sheets are also demonstrated.
\end{abstract}

\section{Introduction}

It is well-known that materials are quite resistant to plastic flow localization when modeled by the classical theory of plasticity with the assumption of smooth yield surface, normality and plastic incompressibility. For example, an initially uniform sheet subject to biaxial tension, modeled by the classical theory for rigid-plastic solids [1], gives unlimited ductility, which is, however, incompatible with experimental observations [2,3]. Deviations from the classical plasticity model can lower the unrealistic ductility. As suggested by Berg [4], the dilatational plastic flow induced by void nucleation and growth is one of significant destabilizing factors.

Gurson $[5,6]$ proposed a set of constitutive relations accounting for plastic dilatancy and pressure sensitivity of yielding due to microvoids for porous materials. To model the plastic flow localization more realistically in accord with experimental observations, Mear and Hutchinson [7] introduced a family of constitutive laws which account for a combination of isotropic and kinematic hardening for porous materials. For purely isotropic hardening cases and/or proportional stressing histories the constitutive behaviors of Mear and Hutchinson's model [7] are identical to those of Gurson's isotropic hardening model $[5,6]$. Mear and Hutchinson [7] found that an increase of the yield surface curvature gives a significant reduction in localization strains and consequently a much smaller void volume fraction is required for shear band formation when compared with those of Gurson's isotropic hardening model $[5,6]$. Recently, Tvergaard [8] incorporated the effect of void nucleation in the model of Mear and Hutchinson [7]. He also found the destabilizing effect of the increased yield surface curvature and in some cases this effect becomes less significant due to the dominant effect of void nucleation.

Pan et al. [9] modified the plastic flow rule proposed by Gurson $[5,6]$ to account for material rate sensitivity. They showed that material rate sensitivity, in contrast to void nucleation and growth, has a retarding effect on shear band formation under both plane strain and axisymmetric tension, and the retarding effect is greater under plane strain tension than under axisymmet- 
ric tension. Needleman and Tvergaard [10] adopted the model of Pan et al. [9] to analyze localized necking in biaxially stretched sheets and observed the same retarding effect of material rate sensitivity. Moreover, Needleman and Tvergaard [10] showed the destabilizing effect of increased plastic potential surface curvature due to kinematic hardening on localized necking in biaxially stretched sheets.

In this paper the effect of rate sensitivity is incorporated into the material model proposed by Mear and Hutchinson [7] and subsequently modified by Tvergaard [8]. Our constitutive relation is similar to that of Becker and Needleman [11] except that they did not consider the effect of void nucleation. Becker and Needleman [11] also found the destabilizing effect of increased plastic potential surface curvature on necking and failure under axisymmetric and plane strain tension through their finite element computations. By using the present material model, the interactions of the effects of void nucleation and growth, material rate sensitivity and plastic potential surface curvature on shear band formation under plane strain and axisymmetric tension, and on localized necking in thin sheets under biaxial stretching are analyzed. Under plane strain and axisymmetric tension, the effect of the enhanced hydrostatic stress due to necking is also studied by using Bridgman's formulae [12] as in Saje et al. [13]. For thin sheets under biaxial stretching, the effect of non-proportional straining paths on localization strains is also investigated.

\section{Constitutive relation}

We employ a Lagrangian formulation and take the initial undeformed configuration as the reference. A material point is identified by Cartesian coordinates $x^{i}$ in the reference state, which is here employed as the convected coordinates. In the current deformed state, the coordinates of a material point, referred to the reference Cartesian base vector, are denoted by $\bar{x}^{i}$. Latin indices range from 1 to 3 and Greek indices range from 1 to 2 . Summation convention is adopted for repeated indices.

The current base vectors $\overline{\mathbf{g}}_{i}$ can be expressed in terms of the deformation gradient tensor $\mathbf{F}$ and the reference base vectors $\mathbf{g}_{j}$ as

$$
\overline{\mathbf{g}}_{i}=F_{. i}^{j} \mathbf{g}_{j} \quad \text { where } \quad F_{. i}^{j}=\frac{\partial \bar{x}^{j}}{\partial x^{i}}
$$

The metric tensors in the reference configuration and in the current configuration are denoted by $g_{i j}$ and $\bar{g}_{i j}$ with the determinants $g$ and $\bar{g}$, respectively. Thus, the Kirchhoff stress tensor $\tau$ and the Cauchy (or true) stress tensor $\sigma$, referring to the deformed coordinates, are related as $\tau=\sqrt{\bar{g} / g} \boldsymbol{\sigma}$.

The covariant components of the Lagrangian strain rate $\dot{\eta}$ are given by

$$
\dot{\eta}_{i j}=\frac{1}{2}\left(F_{. i}^{k} \dot{F}_{k j}+F_{. j}^{k} \dot{F}_{k i}\right)
$$

which can be decomposed into an elastic part $\dot{\eta}_{i j}^{e}$ and a plastic part $\dot{\eta}_{i j}^{p}$ as

$$
\dot{\eta}_{i j}=\dot{\eta}_{i j}^{e}+\dot{\eta}_{i j}^{p}
$$


With the assumption of elastic isotropy, the relation between the elastic Lagrangian strain rate $\dot{\eta}^{e}$ and the Jaumann rate of Cauchy stress $\hat{\sigma}$ is given as

$$
\dot{\eta}_{i j}^{e}=\frac{1}{E}\left[(1+v) \bar{g}_{i k} \bar{g}_{j l}-v \bar{g}_{i j} \bar{g}_{k l}\right] \hat{\sigma}^{k l}
$$

where $E$ is Young's modulus and $v$ is Poisson's ratio. The plastic Lagrangian strain rate $\dot{\eta}^{p}$ can be derived from a plastic potential function and this will be detailed later.

Gurson $[5,6]$ suggested a yield function for porous solids in terms of the void volume fraction $f$, the matrix flow stress $\sigma_{m}$, and the macroscopic stresses. Subsequently, Tvergaard $[14,15]$ included some parameters $q_{i}$ into Gurson's yield function based on the finite element results on plastic flow localization for models having periodically distributed circular cylindrical voids or spherical voids. He suggested $q_{1}=1.5, q_{2}=1$ and $q_{3}=q_{1}^{2}=2.25$. Tvergaard and Needleman [16] also introduced a function $f^{*}(f)$ to account for the loss of stress carrying capacity due to void coalescence. Thus, the modified Gurson's yield function $\phi_{G}$ by the parameters $q_{i}$ and the function $f^{*}(f)$ is

$$
\phi_{G}\left(\sigma, \sigma_{m}, f^{*}\right)=\left(\frac{\sigma_{e}}{\sigma_{m}}\right)^{2}+2 q_{1} f^{*} \cosh \left(\frac{q_{2} \sigma_{. k}^{k}}{2 \sigma_{m}}\right)-1-q_{3} f^{* 2}=0
$$

where $\sigma_{e}$ is an invariant of the macroscopic stresses given by

$$
\sigma_{e}^{2}=\frac{3}{2} \bar{g}_{i k} \bar{g}_{j l} \sigma^{i j} \sigma^{\prime k l}, \quad \sigma^{i j}=\sigma^{i j}-\frac{1}{3} \bar{g}^{i j} \sigma_{. k}^{k}
$$

Here, the tensor $\bar{g}^{i j}$ is the inverse of the metric tensor $\bar{g}_{i j}$. When $q_{1}=q_{2}=q_{3}=1$ and $f^{*}(f)=f$, the modified Gurson's yield function $\phi_{G}$ becomes identical to the original Gurson's yield function. The function $f^{*}(f)$ is given by Tvergaard and Needleman [16] as

$$
f^{*}(f)= \begin{cases}f & \text { for } f \leqslant f_{c} \\ f_{c}+\left(f_{u}^{*}-f_{c}\right)\left(f-f_{c}\right) /\left(f_{f}-f_{c}\right) & \text { for } f>f_{c}\end{cases}
$$

The quantity $f_{u}^{*}$ is defined as the limiting value of $f^{*}(f)$ as the stress carrying capacity goes to zero and $f_{u}^{*}=1 / q_{1}$ from (5). Based on the experimental studies of $[17,18]$ and the numerical analysis of [19], the values of the critical void volume fraction $f_{c}$ and the void volume fraction at the final failure $f_{f}$ were chosen as 0.15 and 0.25 , respectively [16].

Mear and Hutchinson [7] further specified the radius of the yield surface of the matrix material $\sigma_{F}$ as a linear combination of the matrix initial yield stress $\sigma_{y}$ and the matrix flow stress $\sigma_{m}$, and proposed an approximate yield function in the form of $\phi\left(\sigma, \alpha, \sigma_{F}, f\right)=0$. Here, $\alpha$ denotes the current center of the yield surface. The modified yield function of Mear and Hutchinson $\phi$ by the parameters $q_{i}$, the function $f^{*}(f)$ and an appropriate form of $\sigma_{F}$ for rate sensitive materials, is used here as a plastic potential function and it is given by

$$
\phi\left(\sigma, \boldsymbol{\alpha}, \sigma_{F}, f\right)=\left(\frac{\tilde{\sigma}_{e}}{\sigma_{F}}\right)^{2}+2 q_{1} f^{*} \cosh \left(\frac{q_{2} \tilde{\sigma}_{. k}^{k}}{2 \sigma_{F}}\right)-1-q_{3} f^{* 2}=0,
$$


where

$$
\sigma_{F}=(1-b) \frac{\sigma_{y} \sigma_{m}}{g\left(\varepsilon_{m}^{p}\right)}+b \sigma_{m}, \quad \tilde{\sigma}^{i j}=\sigma^{i j}-\alpha^{i j}, \quad \tilde{\sigma}_{e}=\left(\frac{3 \tilde{\sigma}^{i j} \tilde{\sigma}_{i j}^{\prime}}{2}\right)^{1 / 2}, \quad \tilde{\sigma}^{i j}=\tilde{\sigma}^{i j}-\frac{1}{3} \bar{g}^{i j} \tilde{\sigma}_{. k}^{k} .
$$

Here, the parameter $b$ is a constant and has a value between 0 and 1 . The above yield function becomes identical to the modified Gurson's yield function, $\phi_{G}$, of isotropic hardening for $b=1$, while it gives a material model of purely kinematic hardening for $b=0$.

As in Pan et al. [9], the matrix of voided material is now modeled as elastic-viscoplastic. A simple power law for strain rate hardening is used to describe the material viscous behavior:

$$
\dot{\varepsilon}_{m}^{p}=\dot{\varepsilon}_{0}\left[\frac{\sigma_{m}}{g\left(\varepsilon_{m}^{p}\right)}\right]^{1 / m}
$$

where $m$ is the strain rate hardening exponent and $\varepsilon_{m}^{p}$ is the equivalent plastic strain of the matrix. Since $\dot{\varepsilon}_{0}$ is taken here as the reference equivalent plastic strain rate, the function $g\left(\varepsilon_{m}^{p}\right)$ represents the tensile flow stress of the matrix material in the ordinary tensile test at $\dot{\varepsilon}_{m}^{p}=\dot{\varepsilon}_{0}$. For a power-law strain hardening material, the function $g\left(\varepsilon_{m}^{p}\right)$ is given by

$$
\varepsilon_{m}^{p}=\varepsilon_{y}\left[\frac{g\left(\varepsilon_{m}^{p}\right)}{\sigma_{y}}\right]^{1 / N}-\frac{g\left(\varepsilon_{m}^{p}\right)}{E}
$$

where $N$ is the strain hardening exponent, and $\sigma_{y}$ and $\varepsilon_{y}$ are the true yield stress and logarithmic yield strain, respectively, in a uniaxial tensile test conducted at the reference equivalent plastic strain rate $\dot{\varepsilon}_{0}$.

The covariant components of the plastic Lagrangian strain rate $\dot{\eta}^{p}$ are obtained from the plastic potential function $\phi$ as

$$
\dot{\eta}_{i j}^{p}=\dot{\Lambda} \frac{\partial \phi}{\partial \sigma^{i j}}
$$

where $\dot{\Lambda}$ is a proportionality factor which can be determined from the equivalence of plastic work [8]:

$$
\tilde{\sigma}^{i j} \dot{\eta}_{i j}^{p}=(1-f) \sigma_{F} \dot{\varepsilon}_{m}^{p}
$$

Thus, the proportionality factor $\dot{\Lambda}$ becomes

$$
\dot{\Lambda}=\frac{(1-f) \sigma_{F} \dot{\varepsilon}_{m}^{p}}{\tilde{\sigma}^{i j} \frac{\partial \phi}{\partial \sigma^{i j}}}
$$

The increase of void volume fraction arises from the growth of existing voids and from the nucleation of voids. The increment due to growth can be obtained from the plastic incompressibility of the matrix material. For the increment due to nucleation, we here adopt two models: 
one is the plastic strain controlled nucleation model suggested by Gurson $[5,6]$ based on Gurland's experimental data [20] and the other is the stress controlled nucleation model in which void nucleation depends on the maximum stress transmitted across the particle-matrix interface as discussed in Argon and Im [21]. Thus, the increase rate of void volume fraction can be expressed as

$$
\dot{f}=\dot{f}_{\text {growth }}+\dot{f}_{\text {nucleation }}=(1-f) \bar{g}^{i j} \dot{\eta}_{i j}^{p}+A \dot{\varepsilon}_{m}^{p}+B\left[\dot{\sigma}_{m}+\left(\dot{\sigma}_{. k}^{k}\right) / 3\right]
$$

where

$$
\begin{aligned}
& A=\frac{f_{A}}{s \sqrt{2 \pi}} \exp \left[-\frac{1}{2}\left(\frac{\varepsilon_{m}^{p}-\varepsilon_{N}}{s}\right)^{2}\right] \text { for } \dot{\varepsilon}_{m}^{p}>0 \\
& B=\frac{f_{B}}{s \sigma_{y} \sqrt{2 \pi}} \exp \left[-\frac{1}{2}\left(\frac{\sigma_{m}+\sigma_{. k}^{k} / 3-\sigma_{N}}{s \sigma_{y}}\right)^{2}\right] \text { for } \dot{\sigma}_{m}+\left(\dot{\sigma}_{. k}^{k}\right) / 3>0 .
\end{aligned}
$$

Here, $f_{A}$ and $f_{B}$ are the volume fractions of void nucleating particles for the plastic strain controlled nucleation model and the stress controlled nucleation model, respectively, $s$ is the standard deviation, and $\varepsilon_{N}$ and $\sigma_{N}$ are the mean values of the normal distributions.

The evolution equation for the center of plastic potential surface during a plastic deformation is assumed to take the form of

$$
\hat{x}^{i j}=\dot{\mu} \tilde{\sigma}^{i j}, \quad \dot{\mu} \geqslant 0 .
$$

Here, $\hat{\alpha}^{i j}$ represent the contravariant components of the Jaumann rate of $\alpha$. This is a finite strain version of Ziegler's hardening rule [22] that has been used in [7, 23] and the magnitude factor $\dot{\mu}$ will be determined from the consistency equations.

As in [7], under proportional stressing conditions the constitutive behaviors associated with the plastic potential function $\phi$ are taken to be equal to those associated with the modified Gurson's plastic potential function $\phi_{G}$. Here we take [7]

$$
\frac{\sigma^{i j}}{\sigma_{m}}=\frac{\tilde{\sigma}^{i j}}{\sigma_{F}} .
$$

This assumption ensures that $\phi_{G}=0$ when $\phi=0$. In addition, the plastic Lagrangian strain rates are assumed to be equal to each other in the two models based on $\phi=0$ and $\phi_{G}=0$ under proportional stressing conditions. The two consistency equations under plastic loading conditions are

$$
\begin{aligned}
& \dot{\phi}=\frac{\partial \phi}{\partial \sigma^{i j}} \hat{\sigma}^{i j}+\frac{\partial \phi}{\partial \alpha^{i j}} \hat{\alpha}^{i j}+\frac{\partial \phi}{\partial \sigma_{F}} \dot{\sigma}_{F}+\frac{\partial \phi}{\partial f} \dot{f}=0 \\
& \dot{\phi}_{G}=\frac{\partial \phi_{G}}{\partial \sigma^{i j}} \hat{\sigma}^{i j}+\frac{\partial \phi_{G}}{\partial \sigma_{m}} \dot{\sigma}_{m}+\frac{\partial \phi_{G}}{\partial f} \dot{f}=0 .
\end{aligned}
$$


From (12-20), the plastic Lagrangian strain rate $\dot{\eta}^{p}$, the increase rate of void volume fraction $\dot{f}$, the increase rate of the matrix flow stress $\dot{\sigma}_{m}$, and the magnitude factor $\dot{\mu}$ can be determined. Finally, $\hat{\alpha}^{i j}$ is given by

$$
\hat{\alpha}^{i j}=(1-b)\left(\frac{\partial \phi}{\partial \sigma^{k l}} \tilde{\sigma}^{k l}\right)^{-1}\left[\left(1-\frac{\sigma_{y}}{g\left(\varepsilon_{m}^{p}\right)}\right) \frac{\partial \phi}{\partial \sigma^{i j}} \hat{\sigma}^{i j}-\frac{\sigma_{y} \sigma_{m} \dot{\varepsilon}_{m}^{p}}{g\left(\varepsilon_{m}^{p}\right)^{2}} \frac{d g\left(\varepsilon_{m}^{p}\right)}{d \varepsilon_{m}^{p}} \frac{\partial \phi}{\partial \sigma_{F}}\right] \tilde{\sigma}^{i j}
$$

Plugging (4) and (12) into (3) and inverting the equation gives

$$
\hat{\sigma}^{i j}=L^{i j k l} \dot{\eta}_{k l}-\dot{\Lambda} Q^{i j}
$$

where

$$
L^{i j k l}=\frac{E}{1+\nu}\left[\frac{1}{2}\left(\bar{g}^{i k} \bar{g}^{j l}+\bar{g}^{i l} \bar{g}^{j k}\right)+\frac{v}{1-2 v} \bar{g}^{i j} \bar{g}^{k l}\right], \quad Q^{i j}=L^{i j k l} \frac{\partial \phi}{\partial \sigma^{k l}}
$$

We employ the tangent modulus procedure of Peirce et al. [24] to obtain the evolution of the constitutive behavior. We use one of the consistency equations, (20), the void volume fraction increase rate, $(15)$, and the linear interpolation of the matrix plastic strain rate. Then (22) becomes

$$
\hat{\boldsymbol{\sigma}}=\left[\mathbf{L}-\frac{\xi}{1+\xi} \frac{\mathbf{Q p}: \mathbf{L}}{H}\right]: \dot{\eta}-\dot{\Lambda} \frac{\mathbf{Q}}{1+\xi}=\mathbf{L}_{\mathrm{tan}}: \dot{\boldsymbol{\eta}}-\dot{\Lambda} \mathbf{Q}_{\mathrm{tan}}
$$

where

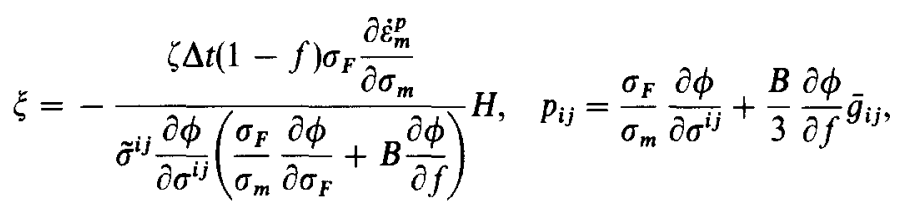

$$
\begin{aligned}
& H=p_{i j} L^{i j k l} \frac{\partial \phi}{\partial \sigma^{k l}}-\frac{\partial \phi}{\partial f}(1-f) \bar{g}^{i j} \frac{\partial \phi}{\partial \sigma^{i j}}-A \frac{\partial \phi}{\partial f} \frac{\tilde{\sigma}^{i j} \frac{\partial \phi}{\partial \sigma^{i j}}}{(1-f) \sigma_{F}} \\
& +\frac{1}{(1-f) \sigma_{F}}\left(\frac{\partial \dot{\varepsilon}_{m}^{p}}{\partial \varepsilon_{m}^{p}}\right)\left(\frac{\partial \dot{\varepsilon}_{m}^{p}}{\partial \sigma_{m}}\right)^{-1}\left(\frac{\sigma_{F}}{\sigma_{m}} \frac{\partial \phi}{\partial \sigma_{F}}+B \frac{\partial \phi}{\partial f}\right) \tilde{\sigma}^{i j} \frac{\partial \phi}{\partial \sigma^{i j}}
\end{aligned}
$$

The parameter $\zeta$ ranges from 0 to 1 , with $\zeta=0$ corresponding to a simple Euler time integration scheme. In (24), two new tensors $\mathbf{L}_{\text {tan }}$ and $\mathbf{Q}_{\tan }$ are defined. Note that the relation between the convected Kirchhoff stress rate $\dot{\tau}$ and the Jaumann rate of Cauchy stress, $\hat{\sigma}$, is given by

$$
i=\sqrt{\bar{g} / g} \hat{\boldsymbol{\sigma}}+\tau \operatorname{tr}(\mathbf{d})-\mathbf{d} \cdot \boldsymbol{\tau}-\boldsymbol{\tau} \cdot \mathbf{d}
$$


where $\mathbf{d}$ is the rate of deformation tensor which is the symmetric part of $\dot{\mathbf{F}} \cdot \mathbf{F}^{-1}$. We also have the identity $\dot{\eta}_{i j}=\overline{\mathbf{g}}_{i} \cdot \mathbf{d} \cdot \overline{\mathbf{g}}_{j}$. Then the constitutive relation between the convected Kirchhoff stress rate and the Lagrangian strain rate can be obtained in the form of

$$
\dot{\tau}^{i j}=\hat{L}_{\mathrm{tan}}^{i j k l} \dot{\eta}_{k l}-\sqrt{\bar{g} / g} \dot{\Lambda} Q_{\mathrm{tan}}^{i j}
$$

where

$$
\hat{L}_{\mathrm{tan}}^{i j k l}=\sqrt{\bar{g} / g} L_{\mathrm{tan}}^{i j k l}+\tau^{i j} \bar{g}^{k l}-\left(\bar{g}^{i k} \tau^{j l}+\bar{g}^{i l} \tau^{j k}+\bar{g}^{j k} \tau^{i l}+\bar{g}^{j l} \tau^{i k}\right) / 2
$$

For the plane stress problems where $i^{3 i}=0$, the constitutive relation becomes

$$
\dot{\tau}^{\alpha \beta}=\left(\hat{L}_{\tan }^{\alpha \beta \gamma \delta}-\frac{\hat{L}_{\tan }^{\alpha \beta 33} \hat{L}_{\tan }^{33 \gamma \delta}}{\hat{L}_{\tan }^{3333}}\right) \dot{\eta}_{\gamma \delta}-\sqrt{\bar{g} / g} \dot{\Lambda}\left(Q_{\tan }^{\alpha \beta}-\frac{\hat{L}_{\tan }^{\alpha \beta 33} Q_{\tan }^{33}}{\hat{L}_{\tan }^{3333}}\right) .
$$

\section{Plastic flow localization analysis}

In the present analyses, a thin planar band with a higher volume fraction of void nucleating particles is assumed to exist in a material element as shown in Fig. 1. Homogeneous deformations inside and outside the band are assumed to occur throughout a deformation history. We consider the element of solid subject to all around displacement boundary conditions, which give plane strain tension, axisymmetric tension and plane stress biaxial deformation. The imposed deformation histories are such that the $x^{1}, x^{2}$ and $x^{3}$ axes remain as the principal directions throughout the deformation histories. The principal values associated with the 1, 2 and 3 directions are denoted by ()$_{I},()_{I I}$ and ( ) III, respectively. In addition, a superscript or subscript ' $b$ ' represents a quantity inside the band, while a superscript or subscript

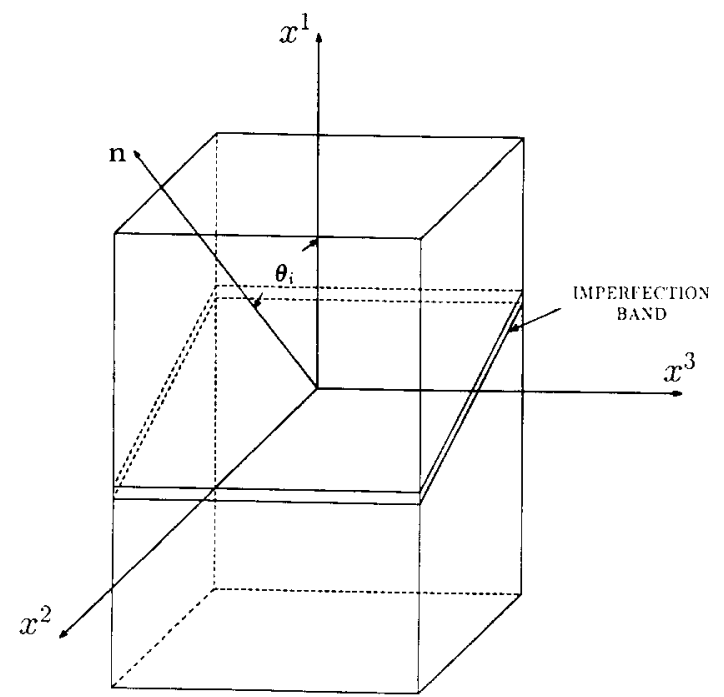

Fig. 1. A solid element having a plane of imperfection. 
' $O$ ' represents a quantity outside the band. Under plane strain tension, $\dot{F}_{\mathrm{III}}^{o}$ is equal to zero and $\dot{F}_{\mathrm{II}}^{o}$ is chosen so that $\dot{\sigma}_{\mathrm{II}}^{o}$ vanishes. Under axisymmetric tension, $\dot{F}_{\mathrm{II}}^{o}=\dot{F}_{\mathrm{III}}^{o}$ with $\dot{\sigma}_{\mathrm{II}}^{o}=\dot{\sigma}_{\mathrm{III}}^{o}=0$. These two deformation histories were originally considered by Yamamoto [25].

We also employ Bridgman's formulae [12] to take account of the effect of enhanced hydrostatic stress due to necking under plane strain tension and axisymmetric tension. For plane strain tension with necking we take $\sigma_{\mathrm{II}}^{o}=\lambda \sigma_{\mathrm{I}}^{o}$ whereas for axisymmetric tension with necking we take $\sigma_{\mathrm{II}}^{o}=\sigma_{\mathrm{III}}^{o}=\lambda \sigma_{\mathrm{I}}^{o}$. Here,

$$
\lambda=\frac{\ln \left(1+\frac{a}{2 r_{c}}\right)}{1+\ln \left(1+\frac{a}{2 r_{c}}\right)},
$$

where $2 a$ is the width (or the diameter) of the minimum section for plane strain tension (or axisymmetric tension), and $r_{c}$ is the radius of curvature of the free surface of the minimum section. Bridgman [12] found a relationship between the ratio $a / r_{c}$ and the axial strain $\varepsilon$ at the minimum section of the form

$$
a / r_{c}= \begin{cases}0 & \text { for } \varepsilon \leqslant N \\ 0.833(\varepsilon-N) & \text { for } \varepsilon>N\end{cases}
$$

where $\varepsilon=\ln \left(A_{i} / A\right)$, with $A_{i}$ being the initial cross sectional area and $A$ being the current cross sectional area at the minimum section. In Bridgman's analysis [12], incompressibility is presumed so that $\varepsilon$ is identified with $\ln \left(F_{1}^{o}\right)$. However, $(31)$ is employed in the present analyses for porous materials.

In simulating thin sheets under biaxial deformation, a plane stress state $\left(\sigma_{\mathrm{III}}^{b}=\sigma_{\mathrm{III}}^{o}=0\right)$ is assumed both inside and outside the band. Under proportional straining conditions, the strain ratio outside the band $\rho\left(=\varepsilon_{1}^{o} / \varepsilon_{2}^{o}\right)$ is prescribed to be a constant throughout a deformation history. Here, $\varepsilon_{1}^{o}=\ln \left(F_{1}^{o}\right)$ and $\varepsilon_{2}^{o}=\ln \left(F_{\mathrm{II}}^{o}\right)$. Under non-proportional straining conditions, the strain ratio $\rho$ is prescribed with two constants of the form as in [10]:

$$
\rho= \begin{cases}\rho_{1} & \text { for } \varepsilon_{1}^{o} \leqslant \varepsilon_{s}^{o} \\ \rho_{2} & \text { for } \varepsilon_{1}^{o}>\varepsilon_{s}^{o}\end{cases}
$$

Two non-proportional straining histories are analyzed: one with $\rho_{1}=-0.5$ (nearly uniaxial tension) and $\rho_{2}=1.0$ (equal-biaxial tension), and the other with $\rho_{1}=1.0$ and $\rho_{2}=0.0$ (in-plane plane strain tension). The variations of localization strains as functions of various $\varepsilon_{s}^{o}$ s are obtained.

As the deformation proceeds, compatibility requires [26, 27]

$$
F_{i j}^{b}=F_{i j}^{o}+c_{i} n_{j}
$$

where $\mathbf{c}$ is a vector denoting the discontinuity across the band and $\mathbf{n}$ is the normal vector to the band in the undeformed state. In addition, equilibrium requires that the nominal tractions be 
continuous over the band interface. Therefore, the equilibrium equation can be given in terms of the first Piola-Kirchhoff stress $\mathbf{s}$ and the normal vector $\mathbf{n}$ as

$$
n_{i} s_{b}^{i j}=n_{i} s_{o}^{i j} \quad \text { with } \quad s^{i j}=\tau^{i k} F_{\cdot k}^{j}
$$

Combining the rate form of the compatibility equation (33) and the rate form of the equilibrium equation (34) with the constitutive relation (27), a set of equations for $\dot{c}_{i}$ is obtained as

$$
\begin{aligned}
& n_{i}\left(\hat{L}_{\mathrm{tan}}^{i k l p} F_{. k}^{j} F_{. l}^{q}+\tau^{i p} g^{j q}\right)_{b} n_{p} \dot{c}_{q}=n_{i}\left(F_{. k}^{j} \sqrt{\bar{g} / g} \dot{\Lambda} Q_{\mathrm{tan}}^{i k}\right)_{b}-n_{i}\left(F_{. k}^{j} \sqrt{\bar{g} / g} \dot{\Lambda} Q_{\mathrm{tan}}^{i k}\right)_{o} \\
& \quad+n_{i}\left(\hat{L}_{\mathrm{tan}}^{i k l p} F_{. k}^{j} F_{. l}^{q}+\tau^{i p} g^{j q}\right)_{o} \dot{F}_{q p}^{o}-n_{i}\left(\hat{L}_{\mathrm{tan}}^{i k l p} F_{. k}^{j} F_{. l}^{q}+\tau^{i p} g^{j q}\right)_{b} \dot{F}_{q p}^{o} .
\end{aligned}
$$

Similarly, a set of equations for $\dot{c}_{\alpha}$ for plane stress biaxial deformations can be obtained.

Given the prescribed deformation histories outside the band, $\dot{F}_{i j}^{o}$, and the initial conditions, the set of equations (35) for $\dot{c}_{i}$ can be solved incrementally to determine the deformation history inside the band. The condition of localization is reached when the ratio of a strain rate inside the band to the counterpart outside the band becomes unbounded.

\section{Numerical results}

In these analyses an additional volume fraction of void nucleating particles, $\Delta f_{A}$ or $\Delta f_{B}$, is assumed to exist inside the band as the material inhomogeneity. Thus, the volume fractions of void nucleating particles inside and outside the band are related by

$$
f_{A}^{b}=f_{A}^{o}+\Delta f_{A}, \quad f_{B}^{b}=f_{B}^{o}+\Delta f_{B}
$$

Both the initial void volume fractions inside and outside the band are taken to be zero. The material under plane strain and axisymmetric tension is specified by $\sigma_{y} / E=0.0033, N=0.1$ and $v=0.3$. Under plane stress biaxial deformation, however, the material parameters are taken as $\sigma_{y} / E=0.001, N=0.2$ and $v=0.3$. For all deformation histories, $\dot{F}_{1}^{o} / F_{1}^{o}$ is prescribed to be $\dot{\varepsilon}_{o}$.

The localization strains are computed for various combinations of $b, m$, and the initial band angle $\theta_{i}$ (as shown in Fig. 1). Under plane strain and axisymmetric tension, the localization strains outside the band $\varepsilon_{1}^{o}$ 's are plotted as functions of both the initial band angle $\theta_{i}$ and the current band angle $\theta_{c}$ in Figs. 2 and 3. In these cases, plastic strain controlled nucleation with $f_{A}^{o}=0.005, \Delta f_{A}=0.005, \varepsilon_{N}=0.3$ and $s=0.1$ is assumed. The minimum of each curve defines the critical localization strain and identifies the most critical initial and current angles of the band containing the material inhomogeneity.

The results of shear band formation under plane strain tension are shown in Fig. 2. As found in [7] and [8], Fig. 2 shows that the localization strains for kinematic hardening cases $(b=0)$ are smaller than those for isotropic hardening cases $(b=1)$. Furthermore, as observed by Pan et al. [9], the localization strains increase as the material becomes more rate sensitive. For the range of $m$ we considered, the retarding effect of material rate sensitivity is a bit more dominant than the destabilizing effect of the increased plastic potential surface curvature. Since we have found a good agreement between the results for rate-dependent materials with $m=0.001$ and 

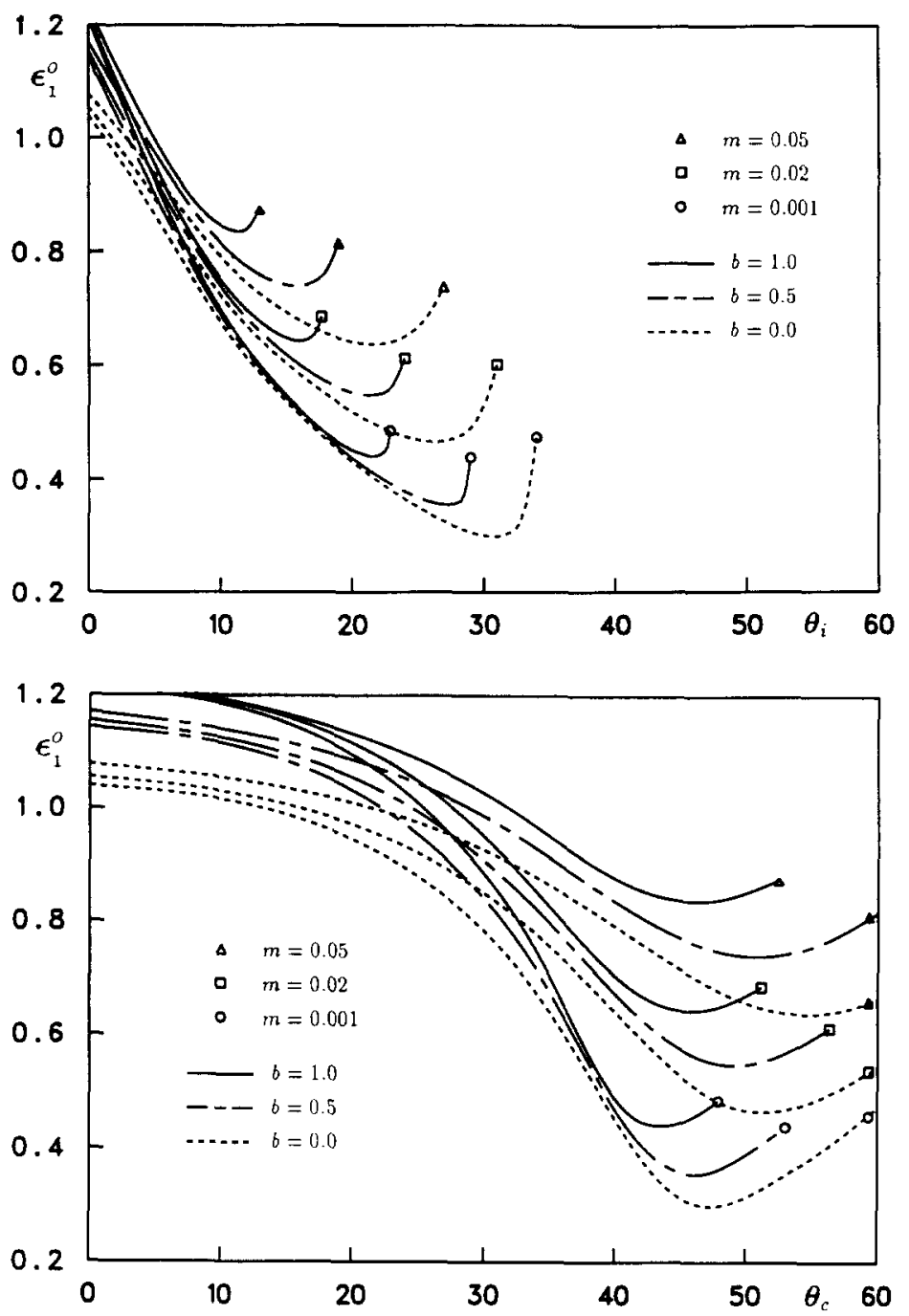

Fig. 2. The localization strains $\varepsilon_{1}^{o}$ s under plane strain tension for $f_{A}^{0}=0.005, \Delta f_{A}=0.005, \varepsilon_{N}=0.3$ and $s=0.1$ as functions of (a) the initial band angle $\theta_{i}$, and (b) the current band angle $\theta_{c}$.

those for the corresponding rate-independent materials, the predictions for $m=0.001$ can be considered as those for rate-independent materials. Figure 2 also shows that the difference of the critical localization strains for isotropic hardening $(b=1)$ and for kinematic hardening $(b=0)$ increases from 0.143 to 0.199 as $m$ increases from 0.001 to 0.05 .

The results of shear band formation under axisymmetric tension are shown in Fig. 3. As shown in Pan et al. [9], the retarding effect of material rate sensitivity under axisymmetric tension is not so strong as that under plane strain tension. However, the destabilizing effect of increased plastic potential surface curvature becomes greater under axisymmetric tension and it is more dominant than the retarding effect of material rate sensitivity. In contrast to the results shown in Fig. 2, the difference of the critical localization strains for isotropic hardening $(b=1)$ and for kinematic hardening $(b=0)$ decreases from 0.557 to 0.514 as $m$ increases from 0.001 to 0.05 . This is due to the fact that, for isotropic hardening with large $m$, the destabilizing effect of high porosity overcomes the retarding effect of material rate sensitivity on localization. 

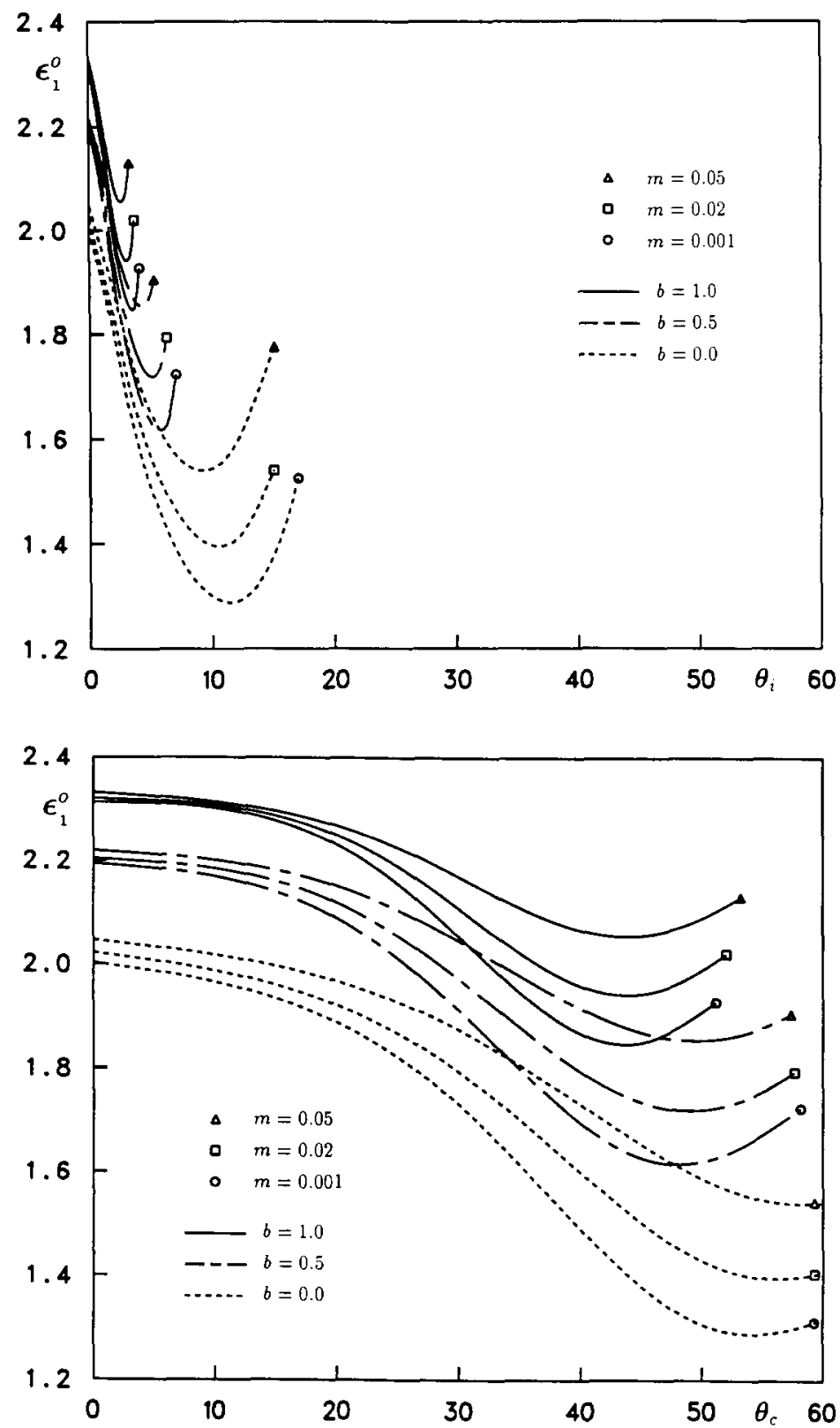

Fig. 3. The localization strains $\varepsilon_{1}^{o}$ 's under axisymmetric tension for $f_{A}^{o}=0.005, \Delta f_{A}=0.005, \varepsilon_{N}=0.3$ and $s=0.1$ as functions of (a) the initial band angle $\theta_{i}$, and (b) the current band angle $\theta_{c}$.

Under both plane strain tension and axisymmetric tension, the critical initial and current band angles for kinematic hardening are larger than those for isotropic hardening. As the material becomes more rate sensitive, the critical initial band angle decreases but the critical current band angle increases. Furthermore, the void volume fractions at localization are smaller for kinematic hardening. For example, for $m=0.02$ shown in Fig. 2, the void volume fraction inside the band at the critical localization strain is 0.032 for $b=0$, whereas the void volume fraction is 0.077 for $b=1$ (these values are taken at $\dot{\eta}_{11}^{b} / \dot{\eta}_{11}^{o}$ about 1000 ). 
We also analyzed the effect of the enhanced hydrostatic stress due to necking on shear band formation under plane strain and axisymmetric tension. The results are plotted in Figs. 4 and 5. These figures show that both the retarding effect of rate sensitivity and the destabilizing effect of increased plastic potential surface curvature are reduced by high triaxiality. As found in [13], the reduction of the localization strains by enhanced triaxiality under axisymmetric tension is more than that under plane strain tension. Under axisymmetric tension, however, the destabilizing effect of the increased plastic potential surface curvature is still more dominant as shown in Fig. 5. For brevity, we choose not to show the variations of localization strains as functions of the current band angles. Similar to the cases without the enhanced hydrostatic stress due to necking, the critical current band angles for kinematic hardening are larger than those for isotropic hardening and these angles increase as $m$ increases. We also have investigated the cases

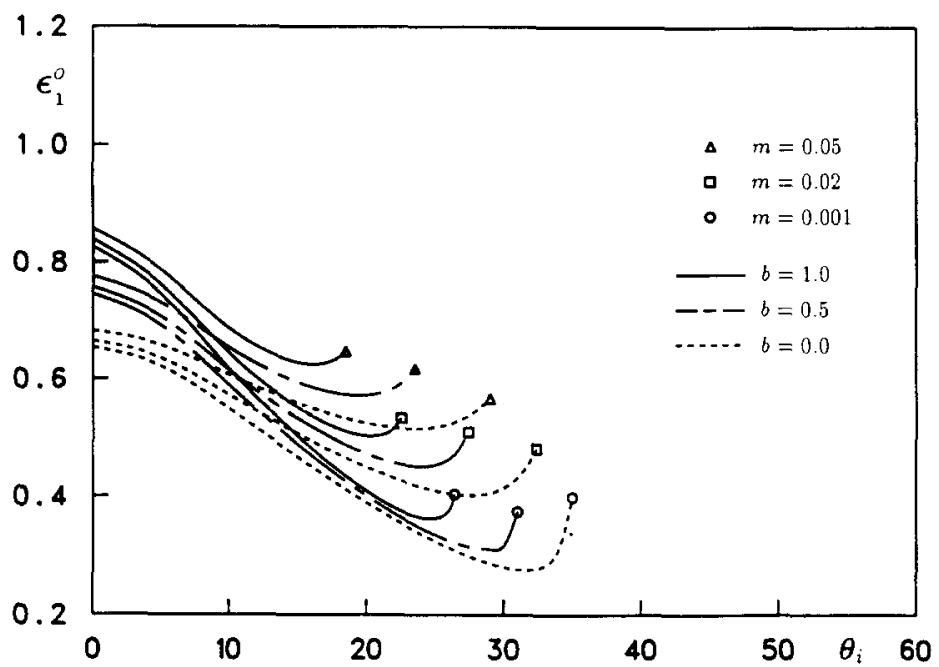

Fig. 4. The localization strains $\varepsilon_{1}^{0}$ 's under plane strain tension with enhanced hydrostatic stress as functions of the initial band angle $\theta_{i}$ for $f_{A}^{o}=0.005, \Delta f_{A}=0.005, \varepsilon_{N}=0.3$ and $s=0.1$.

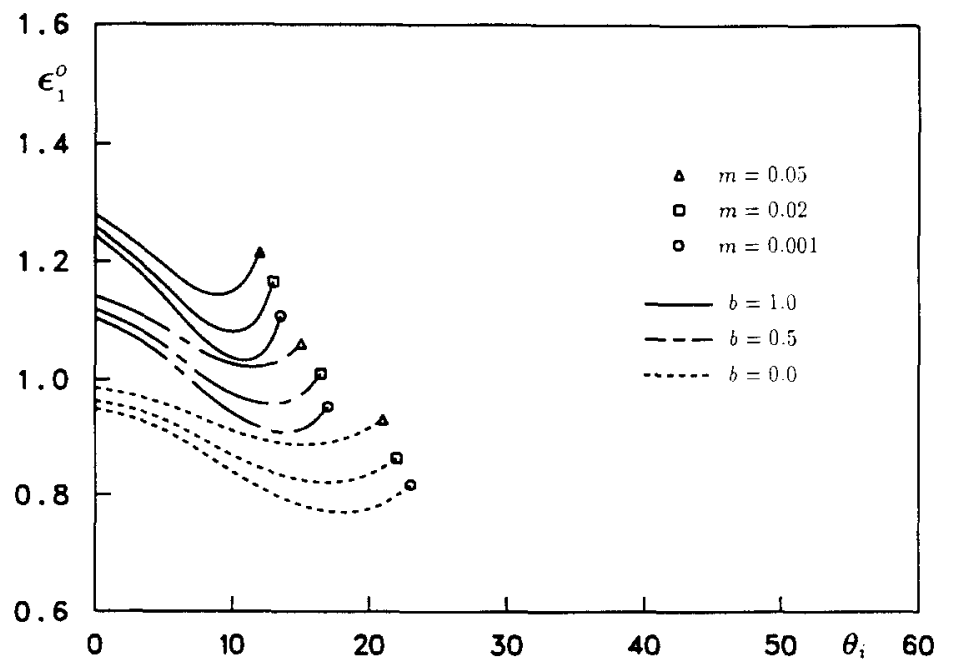

Fig. 5. The localization strains $\varepsilon_{1}^{a}$ 's under axisymmetric tension with enhanced hydrostatic stress as functions of the initial band angle $\theta_{i}$ for $f_{A}^{o}=0.005, \Delta f_{A}=0.005, \varepsilon_{N}=0.3$ and $s=0.1$. 
with a larger inhomogeneity of $\Delta f_{A}=0.015$. As we expected, the results show that a large material inhomogeneity reduces both the effects of rate sensitivity and plastic potential surface curvature as a high triaxiality does.

For plane stress biaxial deformation under proportional and non-proportional straining, we obtained localization strains for various initial band angles and plotted the critical localization strains as the forming limit diagrams shown in Figs. 6, 7 and 8. For the results shown in Figs. 6 and 7, plastic strain controlled nucleation inside and outside the band is assumed with $f_{A}=0.04, \varepsilon_{N}=0.5$ and $s=0.1$. In addition, stress controlled nucleation is assumed inside the band with $\Delta f_{B}=0.01, \sigma_{N}=2.1 \sigma_{y}$ and $s=0.4$. Figure 6 shows the localization strains under proportional straining conditions. For $\rho \geqslant 0$, the critical initial and current band angles are zero. For $\rho<0$, however, the critical initial and current band angles are not zero. For example, for $\rho=-0.5, m=0.05$ and $b=0$, these angles are about $27.5^{\circ}$ and

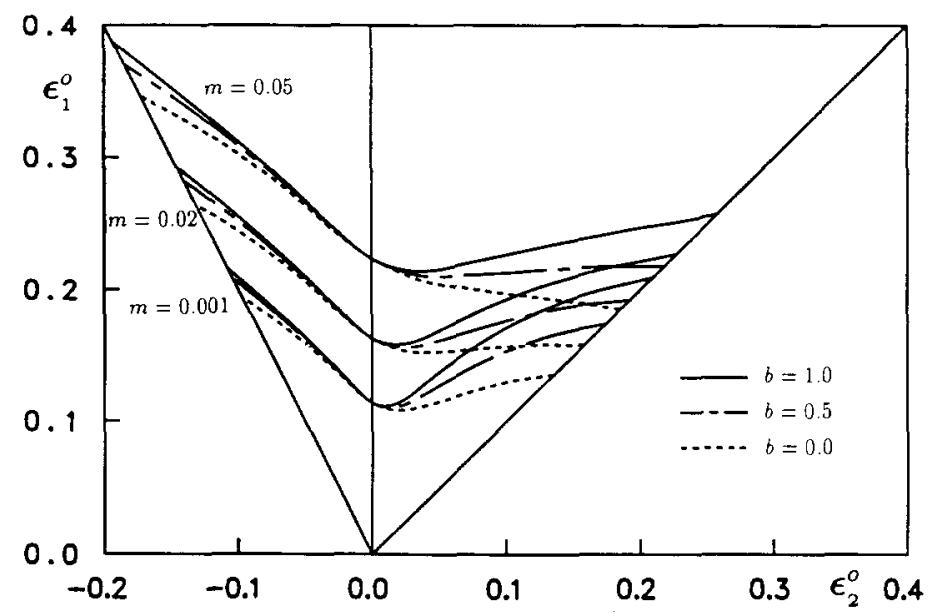

Fig. 6. The forming limit curves under proportional straining for plastic strain controlled nucleation everywhere with $f_{A}^{o}=f_{A}^{b}=0.04, \varepsilon_{N}=0.5$ and $s=0.1$ and stress controlled nucleation inside the band with $\Delta f_{B}=0.01, \sigma_{N}=2.1 \sigma_{y}$ and $s=0.4$

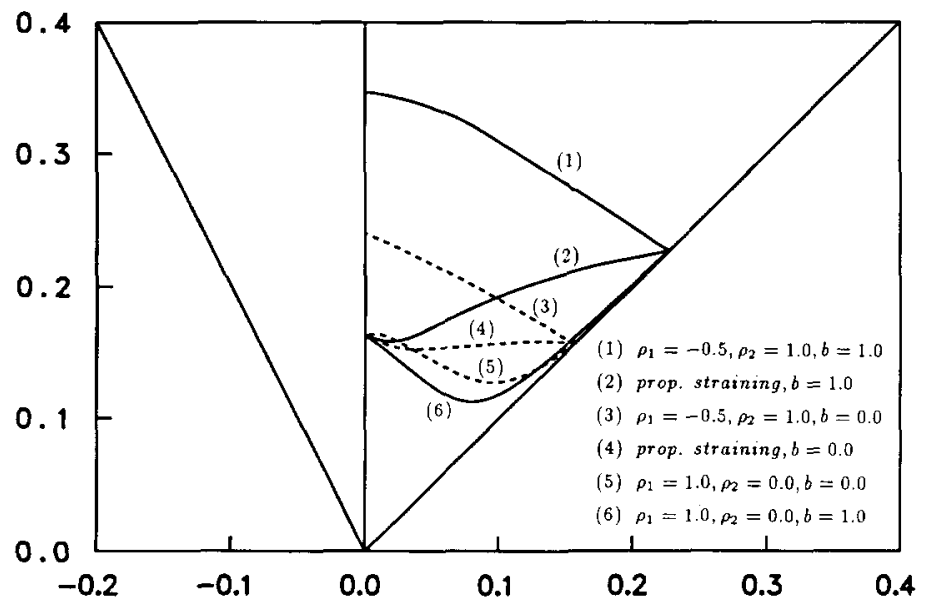

Fig. 7. The forming limit curves for $m=0.02$ under proportional and non-proportional straining for plastic strain controlled nucleation everywhere with $f_{A}^{o}=f_{A}^{b}=0.04, \varepsilon_{N}=0.5$ and $s=0.1$ and additional stress controlled nucleation inside the band with $\Delta f_{B}=0.01, \sigma_{N}=2.1 \sigma_{y}$ and $s=0.4$. 


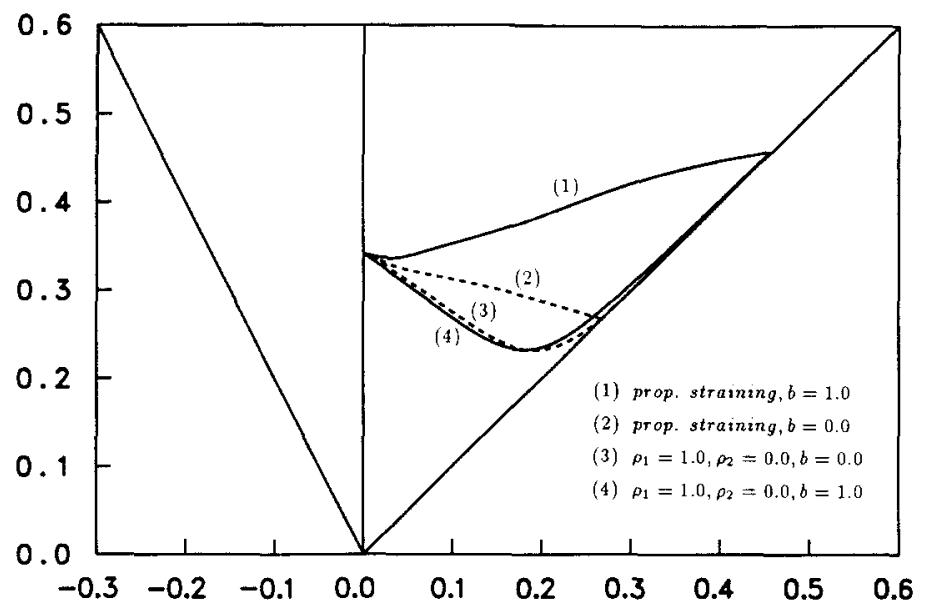

Fig. 8. The forming limit curves for $m=0.02$ under proportional and non-proportional straining for plastic strain controlled nucleation with $f_{A}^{o}=0.04, f_{A}^{b}=0.05, \varepsilon_{N}=0.5$ and $s=0.1$.

$41.1^{\circ}$, respectively. Figure 6 also shows the destabilizing effect of increased plastic potential surface curvature on localized necking formation. The difference of the localization strains for isotropic hardening and for kinematic hardening is almost zero for in-plane plane strain tension $(\rho=0)$ because the loading inside and outside the band is almost proportional. This difference increases as $\rho$ deviates from 0 . The maximum difference occurs at $\rho=1$ where the deformation inside the band changes from biaxial stretching to uniaxial tension when a localized necking is formed.

Figure 7 shows the effect of straining paths on localization strains. A comparison of these curves indicates that in general pre-biaxial straining results in lower localization strains, but pre-uniaxial straining results in higher localization strains than those of the corresponding proportional straining cases. However, for the kinematic hardening cases, the localization strains of curve (5) for pre-biaxial straining are higher than those of curve (4) for proportional straining only for small values of $\varepsilon_{2}^{o}$ (or $\varepsilon_{s}^{o}$ ). These results essentially agree with those of [10] where a rate dependent kinematic hardening plasticity model was used to investigate the straining path effect on necking in thin sheets with thickness imperfections. Figure 7 also shows that due to pre-biaxial straining the localization strains of curve (5) for kinematic hardening are higher than those of curve (6) for isotropic hardening for $\varepsilon_{2}^{o}$ (or $\varepsilon_{s}^{o}$ ) up to 0.130 . The hump of curve (5) over curve (4) and the higher localization strains of curve (5) than those of curve (6) result from the stress controlled nucleation model with a low mean value $\sigma_{N}$. When we employ the plastic strain controlled nucleation model only with $f_{A}^{o}=0.04, \Delta f_{A}=0.01$, as shown in Fig. 8 , pre-biaxial straining results in almost the same localization strains of curve (4) for isotropic hardening as those of curve (3) for kinematic hardening for $\varepsilon_{2}^{o}$ (or $\varepsilon_{s}^{o}$ ) up to 0.183 .

\section{Conclusions}

We have analyzed the shear band formation under plane strain and axisymmetric tension, and the localized necking in biaxially deformed sheets. We have shown that material rate sensitivity, plastic potential surface curvature, high triaxiality and straining path have strong influences on plastic flow localization. As the material becomes more rate-sensitive, the difference of the 
critical localization strains for isotropic hardening and for kinematic hardening increases under plane strain tension, but it decreases a bit under axisymmetric tension. The retarding effect of material rate sensitivity under axisymmetric tension is not so strong as that under plane strain tension. Under axisymmetric tension the destabilizing effect of the increased plastic potential surface curvature becomes more significant than the retarding effect of rate sensitivity. With a high triaxiality due to necking or a large material inhomogeneity, both the retarding effect of rate sensitivity and the destabilizing effect of increased plastic potential surface curvature are mitigated, and the difference of the critical localization strains for isotropic hardening and for kinematic hardening is also reduced.

For proportional straining cases, the slope of the forming limit curves becomes smaller for kinematic hardening and/or for high rate hardening exponent. It is noted that, in general, pre-uniaxial straining results in higher localization strains, but pre-biaxial straining results in lower localization strains than those under proportional straining. For pre-biaxial straining with a small prestrain $\varepsilon_{s}^{o}$, however, the stress controlled nucleation model results in higher localization strains for kinematic hardening than those for isotropic hardening but the plastic strain controlled nucleation model results in almost the same values for both isotropic and kinematic hardening. This means that, for pre-biaxial straining, kinematic hardening results in higher ductility than isotropic hardening when the stress controlled nucleation model is applied. Furthermore, in all the cases of plane strain tension, axisymmetric tension and plane stress biaxial deformation, the critical initial and current band angles for kinematic hardening are larger than those for isotropic hardening. Moreover, the critical initial band angle decreases even though the critical current band angle increases as the material becomes more rate sensitive.

\section{Acknowledgement}

The support of this work by a grant from the University Research Program of Ford Motor Company and a Material Research Group grant funded by the National Science Foundation under grant no. DMR-8708405 at The University of Michigan is gratefully acknowledged. Helpful discussions of this work with Prof. A. Needleman of Brown University, Prof. V. Tvergaard of the Technical University of Denmark, and Prof. J.W. Hutchinson of Harvard University are also appreciated.

\section{References}

1. R. Hill, Journal of Mechanics and Physics of Solids 1 (1952) 19-30.

2. M. Azrin and W.A. Backofen, Metallurgical Transactions 1 (1970) 2857-2865.

3. A.K. Ghosh and S.S. Hecker, Metallurgical Transactions 5 (1974) 2161-2164.

4. C.A. Berg, Inelastic Behavior of Solids, M.F. Kanninen et al. (eds.), McGraw-Hill, New York (1970) 171-209.

5. A.L. Gurson, Plastic Flow and Fracture Behavior of Ductile Materials Incorporating Void Nucleation, Growth and Interaction, Ph.D. thesis, Brown University, Providence, Rhode Island (1975).

6. A.L. Gurson, Journal of Engineering Materials and Technology 99 (1977) 2-15.

7. M.E. Mear and J.W. Hutchinson, Mechanics of Materials 4 (1985) 395-407.

8. V. Tvergaard, Journal of Mechanics and Physics of Solids 35 (1987) 43-60.

9. J. Pan, M. Saje and A. Needleman, International Journal of Fracture 21 (1983) 261-278. 
10. A. Needleman and V. Tvergaard, Mechanical Behaviour of Materials - IV, J. Carlsson and N.G. Ohlson, (eds.), Pergamon Press (1984) 51-65.

11. R. Becker and A. Needleman, Journal of Applied Mechanics 53 (1986) 491-499.

12. P.W. Bridgman, Studies in Large Plastic Flow and Fracture, McGraw-Hill (1952).

13. M. Saje, J. Pan and A. Needleman, International Journal of Fracture 19 (1982) 163-182.

14. V. Tvergaard, International Journal of Fracture 17 (1981) 389-407.

15. V. Tvergaard, International Journal of Fracture 18 (1982) 237-252.

16. V. Tvergaard and A. Needleman, Acta Metallurgica 32 (1984) 157-169.

17. L.M. Brown and J.D. Embury, Proceedings 3rd International Conference on Strength of Metals and Alloys, Institute of Metals, London (1973) 164-169.

18. S.H. Goods and L.M. Brown, Acta Metallurgica 27 (1979) 1-15.

19. H. Andersson, Journal of Mechanics and Physics of Solids 25 (1977) 217-233.

20. J. Gurland, Acta Metallurgica 20 (1972) 735-741.

21. A.S. Argon and J. Im, Metallurgical Transactions 6A (1975) 839-851.

22. H. Ziegler, Quarterly of Applied Mathematics 17 (1959) 55-65.

23. V. Tvergaard, International Journal of Mechanical Sciences 20 (1978) 651-658.

24. D. Peirce, C.F. Shih and A. Needleman, Computers \& Structures 18 (1984) 875-887.

25. H. Yamamoto, International Journal of Fracture 11 (1978) 347-365.

26. R. Hill, Journal of Mechanics and Physics of Solids 10 (1962) 1-16.

27. J.R. Rice, Proceedings 14th International Conference on Theoretical and Applied Mechanics, Delft, North-Holland, W.T. Koiter (ed.), 1 (1976) 207-220. 